EESTI NSV TEADUSTE AKADEEMIA TOIMETISED. XII KÖIDE

FUUSIKA-, MATEMAATIKA- JA TEHNIKATEADUSTE SEERIA, 1963, NR. 1

ИЗВЕСТИЯ АКАДЕМИИ НАУК ЭСТОНСКОН ССР. ТОМ ХІІ

СЕРИЯ ФИЗИКО-МАТЕМАТИЧЕСКИХ И ТЕХНИЧЕСКИХ НАУК. 1963, № 1

\title{
О РАСЧЕТНЫХ МОДЕЛЯХ УПРУГИХ ПЛАСТИНОК ДЛЯ ДИНАМИЧЕСКИХ ЗАДАЧ
}

\author{
Л. АИнолА, \\ кандидат физико-математических наук
}

Решение задач распространения упругих волн в пластинках при помощи уравнений трехмерной теории упругости связано с большими математическими трудностями, поэтому при их решении приходится нспользовать простые модели, полученные путем приведения трехмерных уравнений теории упругости к двумерным уравнениям пластинки. Для аңтисимметричных задач простейшую модель пластинки представляет теория Кирхгофа, для симметричных задач - теория плоского напряженного состояния. В последнее время различными методами приведения получен ря,д вариантов т. н. уточненных уравнений пластинок $[1-8]$.

В настоящей статье дается некоторое обобщение этих уточненных теорий. В пер вом разделе работы, предполагая заданными изменения перемещений и напряжений по толщине шластинки, выводятся уравнения пластинки шестого порядка в общей форме. В следующих разделах рассматриваются теории, где это изменение выбрано соответственно частному решению трехмерной теорин упругости. Этот случай может представлять интерес при исследовании переходных волновых процессов, вызванных нагрузкой определенной частоты. В конце статьи выводятся уравнения пластинки впредельном случае, если распределение перемещений аппроксимируется в виде, получаемом при распространении бесконечно длинных элементарных волн по первой форме движения. Пля антисимметричных задач выведенные уравнения пластинки совпадают с уравнениями типа Тимошенко $\left[{ }^{2}\right]$, если в них значение коэффициента $\kappa^{2}$ взять равным 5/6. Для симметричных задач выведенные уравнения существенно отличаются от соответствующих уравнений Кейна-Миндлина [].

\section{1. Уравнения пластинки}

Рассмотрим пластинку постоянной толщины $2 h$, срединная поверхность которой совпадает с координатной плоскостью $x^{1} x^{2}$. Предположим, что пластинка не нагружена на поверхностях $x^{3} \doteq z= \pm h$.

Уравнения пластинки выводим из вариационного принципа, динамики трехмерной теории упругости, которому даем такую форму, где, кроме перемещений $u_{i}$, независимыми варьируемыми функциями являлись бы и напряжения $\sigma^{\text {i3 }}$ и деформации $\varepsilon_{33}$ :

$$
\begin{aligned}
& \delta\left\{\int _ { t _ { 0 } V } ^ { t _ { 1 } } \int _ { 2 } \left(\frac{1}{2} \sigma^{\alpha \beta} \varepsilon_{\alpha \beta}-\frac{1}{2 \mu} \widehat{\sigma}^{\alpha 3} \widehat{\sigma}_{\alpha 3}-\widehat{\sigma}^{33} \widehat{\varepsilon}_{33}-\right.\right. \\
& \left.-\nabla_{3} \widehat{\sigma}^{3 i} u_{\mathrm{i}}+\widehat{\sigma}^{\alpha 3} \nabla_{\alpha} u_{3}-\frac{1}{2} \varrho \dot{u}_{\mathrm{i}} \dot{u}^{i}\right) d V d t+
\end{aligned}
$$




$$
\begin{gathered}
\left.+\int_{t_{0}}^{t_{1}} \int_{S_{1,2}} \widehat{\sigma^{\mathrm{i} 3}} u_{\mathrm{i}} d S d t-\int_{t_{0}}^{t_{1}} \int_{C} Q^{\mathrm{i}} u_{\mathrm{i}} d C d t\right\}=0 \\
(i=1,2,3 ; \alpha, \beta=1,2)
\end{gathered}
$$

Здесь $u_{1}$ - варьируемые перемещения, удовлетворяющие геометрическим граничным условиям; $\sigma^{\alpha \beta}$ - напряжения, выраженные через перемещения $u_{\mathrm{i}}$, причем $\nabla_{3} u_{3}$ заменено $\widehat{\varepsilon_{33}} ; \widehat{\sigma}^{\mathrm{i3}}-$ варьируемые напряжения, удовлетворяющие статическим граничным условиям на поверхностях $z= \pm h$ пласткнки; $\hat{\varepsilon}_{33}-$ варьируемые деформации; $S_{1,2}-$ поверхности $z= \pm h$ пластинки; $C-$ контурная поверхность пластинки; $Q^{i}-$ внешние силы, заданные на контурной поверхности $C$. Точка обозна чаег производную по времени $t$.

Аппроксимируем варьируемые величины следующим образом:

$$
\begin{gathered}
u_{\alpha}=\varphi_{\alpha}\left(x^{\gamma}, t\right) \cdot \psi_{1}(z), u_{3}=w\left(x^{\gamma}, t\right) \cdot \psi_{2}(z), \\
\hat{\sigma}^{\alpha 3}=\tau^{\alpha}\left(x^{\gamma}, t\right) \cdot \psi_{3}(z), \widehat{\sigma}^{33}=\tau^{3}\left(x^{\gamma}, t\right) \cdot \psi_{4}(z), \\
\hat{\varepsilon}_{33}=e\left(x^{\gamma}, t\right) \cdot \psi_{5}(z),
\end{gathered}
$$

где $\psi_{s}(s=1,2, \ldots, 5)$ - заданные функции, удовлетворяющие условиям

$$
\psi_{3}( \pm h)=\psi_{4}( \pm h)=0
$$

Введем обозначения

$$
H^{\alpha \beta}=\int_{-h}^{h} \sigma^{\alpha \beta} \psi_{1} d z
$$

11

$$
a_{s t}=\int_{-h}^{h} \psi_{s} \psi_{t} d z, \quad a_{s t}^{\prime}=\int_{-h}^{h} \frac{d \psi_{s}}{d z} \psi_{t} d z
$$

Из вариационного принципа (1.1) по аппроксимации (1.2) вытекают уравнения равновесия пластинки

$$
\begin{aligned}
& \nabla_{\beta} H^{\alpha \beta}+a_{31}^{\prime} \tau^{\alpha}-\varrho a_{11} \ddot{\varphi}^{\alpha}=0, \\
& a_{23} \nabla_{\alpha} \tau^{\alpha}+a_{42}^{\prime} \tau^{3}-\varrho a_{22} \ddot{w}=0
\end{aligned}
$$

и соотношения

$$
\begin{gathered}
a_{33} \tau^{\alpha}-\mu\left(a_{13}^{\prime} \varphi^{\alpha}+a_{23} \nabla^{\alpha} w\right)=0, \\
a_{45} \tau^{3}-(\lambda+2 \mu) a_{55} e-\lambda a_{15} \nabla^{\alpha} \varphi_{\alpha}=0, \\
a_{45} e-a_{24}^{\prime} w=0,
\end{gathered}
$$

тде

$$
H^{\alpha \beta}=a_{11}\left[\mu\left(\nabla^{\alpha} \varphi^{\beta}+\nabla^{\beta} \varphi^{\alpha}\right)+\lambda g^{\alpha \beta} \nabla^{\gamma} \varphi_{\gamma}\right]+\lambda a_{15} g^{\alpha \beta} e .
$$


Граничные условия на контуре $C$ пластинки получаются в следующем виде:

$$
H^{\alpha \beta} n_{\alpha}-R^{\beta}=0, \quad a_{23} \tau^{\alpha} n_{\alpha}-P=0
$$

и

где

$$
\varphi_{\alpha}=\bar{\varphi}_{\alpha}, \quad w=\bar{w},
$$

$$
R^{\alpha}=\int_{-h}^{h} Q^{\alpha} \psi_{1} d z, \quad P=\int_{-h}^{h} Q^{3} \psi_{2} d z .
$$

При помощи соотношений (1.6) и (1.7) уравнения равновесия (1.5) могут быть приведены к виду

$$
\begin{gathered}
(\lambda+\mu) \nabla^{\alpha} \nabla^{r} \varphi_{\gamma}+\mu \nabla^{2} \varphi^{\alpha}+\kappa_{1} \varphi^{\alpha}-\varrho \ddot{\varphi}^{\alpha}+\kappa_{2} \nabla^{\alpha} w=0, \\
\nabla^{r} \varphi_{7}+\kappa_{3} \nabla^{2} w+\kappa_{4} w-\varrho \kappa_{5} \ddot{w}=0 .
\end{gathered}
$$

Здесь

$$
\begin{aligned}
& \kappa_{1}=-\mu \frac{a_{13}^{\prime 2}}{a_{11} a_{33}}, \quad \kappa_{2}=\lambda \frac{a_{15} a_{24}^{\prime}}{a_{11} a_{45}}+\mu \frac{a_{31}^{\prime} a_{23}}{a_{11} a_{33}}, \\
& \kappa_{3}=\mu \frac{a_{23}^{2}}{a_{11} a_{33} \kappa_{6}}, \quad \kappa_{4}=-(\lambda+2 \mu) \frac{a_{24}^{\prime} a_{42}^{\prime} a_{55}}{a_{11} a_{45}^{2} \kappa_{6}}, \\
& \kappa_{5}=\frac{a_{22}}{a_{11} \kappa_{6}}, \quad \kappa_{6}=-\lambda \frac{a_{15}}{a_{1 !}}-\mu \frac{a_{31}^{\prime} a_{23}}{a_{11} a_{33}} .
\end{aligned}
$$

Исключая из уравнений (1.10) функции $\varphi_{\alpha}$, получаем уравнение

$$
\left\{\left[(\lambda+2 \mu) \nabla^{2}+\kappa_{1}-\varrho \partial_{t}^{2}\right]\left[\kappa_{3} \nabla^{2}+\kappa_{4}-\varrho \kappa_{5} \partial_{t}^{2}\right]-\kappa_{2} \nabla^{2}\right\} w=0 .
$$

Функции $\varphi_{x}$ могут быть представлены в виде суммы

$$
\varphi_{\alpha}=\varphi_{\alpha 1}+\varphi_{\alpha 2},
$$

где $\varphi_{a 1}$ - частное рсшение системы $(1.10)$ и $\varphi_{a 2}$ - решение соответствуюшей однородной системы. Если

$$
\varphi_{\alpha 2}=c_{\alpha} \cdot \nabla_{\beta} \Phi
$$

( $c_{\alpha \beta}-$ дискриминантный тензор), то эту однородную систему можно привести к уравнению

$$
\mu \nabla^{2} \Phi+\kappa_{1} \Phi-\varrho \ddot{\Phi}=0
$$

\section{2. Уравнения для антисимметричных задач}

Если выбрать аппроксимируемые функции $\psi_{1}, \psi_{4}, \psi_{5}$ нечетными, а функции $\psi_{2}, \psi_{3}$ - четными, то (1.12), (1.15) будут уравнения пластинки для антисимметричных задач.

Рассмотрим подробнее один частный случай. Именно, предположим, что функции $\psi_{s}$ выбраны в таком виде, как они получаются по точной теории при распространении элементарных волн определенных частот. 
Если $p-$ волновое число, $\omega-$ круговая частота волны и если обозначить

$$
\alpha=\sqrt{p^{2}-\frac{\varrho \omega^{2}}{\lambda+2 \mu}}, \beta=\sqrt{p^{2}-\frac{Q \omega^{2}}{\mu}}
$$

то частное решение трехмерной теории упругости можем представить в виде $\psi_{s} b_{s} e^{i(p x+\infty i)}$, где $b_{1}=b_{3}=1, b_{2}=b_{4}=b_{5}=i$ и

$\psi_{1}=(2 \operatorname{sh} \beta h)^{-1}\left[2 p^{2} \operatorname{sh} \beta h \operatorname{sh} \alpha z-\left(p^{2}+\beta^{2}\right) \operatorname{sh} \alpha h \operatorname{sh} \beta z\right]$,

$\Psi_{2}=-p(2 \beta \operatorname{sh} \beta h)^{-1}\left[2 \alpha \beta \operatorname{sh} \beta h \operatorname{ch} \alpha z-\left(p^{2}+\beta^{2}\right) \operatorname{sh} \alpha h \operatorname{ch} \beta z\right]$,

$\psi_{3}=(2 \beta \operatorname{sh} \beta h)^{-1}\left[4 p^{2} \alpha \beta \operatorname{sh} \beta h \operatorname{ch} \alpha z-\left(p^{2}+\beta^{2}\right)^{2} \operatorname{sh} \alpha h \operatorname{ch} \beta z\right]$,

$\psi_{4}=-\mu p\left(p^{2}+\beta^{2}\right)(\operatorname{sh} \beta h)^{-1}(\operatorname{sh} \beta h \operatorname{sh} \alpha z-\operatorname{sh} \alpha h \operatorname{sh} \beta z)$,

$\psi_{5}=-p(2 \operatorname{sh} \beta h)^{-1}\left[2 \alpha^{2} \operatorname{sh} \beta h \operatorname{sh} \alpha z-\left(p^{2}+\beta^{2}\right) \operatorname{sh} \alpha h \operatorname{sh} \beta z\right]$,

причем предполагается, что величины $p, \alpha$ и $\beta$ удовлетворяют уравнению Лэмба

$$
\frac{\text { th } \alpha h}{\text { th } \beta h}=\frac{4 p^{2} \alpha \beta}{\left(p^{2}+\beta^{2}\right)^{2}} \text {. }
$$

Имея заданную частоту $\omega$ при конкретной форме движения, коэффициенты уравнений пластинки (1.12) и (1.15) легко определяются по соотношениям $(2.2),(1.4)$ и (1.11).

Особый интерес представляет предельный случай, где коэффициенты в уравнениях пластинки выбраны соответственно распространению бесконечно длинных волн по первой форме движения. Из уравнения Лэмба (2.3) для этого случая вытекает, что

$$
\begin{aligned}
& \alpha=p\left[1-\frac{2 \mu(\lambda+\mu)}{3(\lambda+2 \mu)^{2}} p^{2} h^{2}\right], \\
& \beta=p\left[1-\frac{2}{3} \frac{\lambda+\mu}{\lambda+2 \mu} p^{2} h^{2}\right] .
\end{aligned}
$$

Сохраняя в функциях $\psi_{s}$ только члены с наименьшими степенями по $p$ и учитывая соотношения (2.4), получаем

$$
\psi_{s}=c_{s} \vartheta_{s},
$$

где $c_{s}$ - постоянные числа и $\vartheta_{1}=p^{5} z, \quad \vartheta_{2}=p^{4}, \quad \vartheta_{3}=p^{7}\left(z^{2}-h^{2}\right)$, $\vartheta_{4}=p^{8} z\left(z^{2}-h^{2}\right), \quad \vartheta_{5}=p^{6} z$.

Соответствующие коэффициенты $a_{s t}$ и $a_{s t}^{\prime}$ будут

причем

$$
a_{s t}=c_{s} c_{t} b_{s t}, \quad a_{s t}^{\prime}=c_{s} c_{t} b_{s t}^{\prime},
$$

$$
\begin{gathered}
b_{11}=\frac{2}{3} p^{10} h^{3}, \quad b_{15}=\frac{2}{3} p^{11} h^{3}, \quad b_{22}=2 p^{10} h \\
b_{23}=-\frac{4}{3} p^{11} h^{3}, \quad b_{33}=\frac{16}{15} p^{14} h^{5}, \quad b_{45}=b_{24}^{\prime}=-b_{42}^{\prime}=-\frac{4}{15} p^{14} h^{5}, \\
b_{55}=\frac{2}{3} p^{12} h^{3}, \quad b_{31}^{\prime}=-b_{13}^{\prime}=-\frac{4}{3} p^{12} h^{3} \\
b_{24}^{\prime}=-b_{42}^{\prime}=-\frac{4}{15} \frac{\lambda}{\mu} p^{14} h^{5}
\end{gathered}
$$


Подставляя найденные коэффициенты $a_{s t}$ и $a_{s t}^{\prime}$ в уравнения (1.5) и в соотношения (1.6), видим, что в уравнениях (1.5) член $a_{42}^{\prime} \tau^{3}$ и в соот. ношениях (1.6) член $a_{45} \tau^{3}$ являются малыми более высокого порядка, поэтому ими нужно пренебречь ${ }^{*}$. Из соотношений $(2.6)$ и (1.6) тогда следует, что

$$
e=p^{-1} \nabla^{a} \varphi_{\alpha} .
$$

Если это ввести в выражение моментов (1.7) и учесть соотношение (2.6), получаем, что

$$
H^{\alpha \beta}=\frac{E a_{11}}{2\left(1-v^{2}\right)}\left[\nabla^{a} \varphi^{\beta}+\nabla^{\beta} \varphi^{\alpha}+v c^{\alpha \gamma} c^{3 \hat{\jmath}}\left(\nabla_{\gamma} \varphi_{i}+\nabla_{i} \varphi_{i}\right)\right] .
$$

Далее. из уравнений (1.5) при помощи соотношений (2.8) и (1.6) вытекают уравнения пластинки

$$
\begin{gathered}
{\left[\left(\frac{E h^{3}}{1-v^{2}} \nabla^{2}-\rho h^{3} \partial_{t}^{2}\right)\left(\nabla^{2}-\frac{6}{5} \frac{\varrho}{\mu} \partial_{t}^{2}\right)+3 \rho h \partial_{t}^{2}\right] w=0,} \\
\left(\mu \nabla^{2}-\frac{5}{2} \mu h^{-2}-\rho \partial_{t}^{2}\right) \Phi=0 .
\end{gathered}
$$

Эти уравнения совпадают с уравнениями типа Тимошенко [1, 2, 4 , если в последних значением коэффициента $k^{2}$ взять $\frac{5}{6}$. Это значение коэффициента обусловлено параболическим распределением тангенциальных напряжений $\sigma^{\alpha 3}$.

\section{3. Уравнения для симметричных задач}

Уравнения пластинки для симметричных задач получаются, если аппроксимировать $\psi_{1}, \psi_{4}, \psi_{5}$ четными и $\psi_{2}, \psi_{3}$ нечетными функциями.

В симметричном случае аппроксимация по элементарной волне точной теорин будет

$\psi_{1}=(2 \operatorname{ch} \beta h)^{-1}\left[2 p^{2} \operatorname{ch} \beta h \operatorname{ch} \alpha z-\left(p^{2}+\beta^{2}\right) \operatorname{ch} \alpha h \operatorname{ch} \beta z\right]$,

$\psi_{2}=-p(2 \beta \operatorname{ch} \beta h)^{-1}\left[2 \alpha \beta \operatorname{ch} \beta h \operatorname{sh} \alpha z-\left(p^{2}+\beta^{2}\right) \operatorname{ch} \alpha h \operatorname{sh} \beta z\right]$,

$\psi_{3}=\mu(2 \beta \operatorname{ch} \beta h)^{-1}\left[4 p^{2} \alpha \beta \operatorname{ch} \beta h \operatorname{sh} \alpha z-\left(p^{2}+\beta^{2}\right)^{2} \operatorname{ch} \alpha h \operatorname{sh} \beta z\right]$,

$\psi_{4}=-\mu p\left(p^{2}+\beta^{2}\right)(\operatorname{ch} \beta h)^{-1}(\operatorname{ch} \beta h \operatorname{ch} \alpha z-\operatorname{ch} \alpha h \operatorname{ch} \beta z)$.

$\psi_{5}=-p(2 \operatorname{ch} \beta h)^{-1}\left[2 \alpha^{2} \operatorname{ch} \beta h \operatorname{ch} \alpha z-\left(p^{2}+\beta^{2}\right) \operatorname{ch} \alpha h \operatorname{ch} \beta z\right]$,

где величины $p, \alpha, \beta$ связаны уравнением Лэмба

$$
\text { th } \alpha h=\frac{\left(p^{2}+\beta^{2}\right)^{2}}{4 p^{2} \alpha \beta} .
$$

- Этому обстоятельству соответствует гипотеза $\sigma^{33}=0$ при обычном выводе теории типа Тимошенко. 
В предельном случае, при бесконечно длинных волнах и при первой форме движения из (3.2) вытекает, что

$$
\alpha=\frac{\lambda}{\lambda+2 \mu} p, \quad \beta=\sqrt{\frac{3 \lambda+2 \mu}{\lambda+2 \mu}} i p
$$

Функции $\psi_{s}$ в этом случае таковы -

$$
\psi_{s}=d_{s} \hat{\theta}_{s},
$$

где $d_{s}$ - постоянные числа и $\vartheta_{1}=p^{2}, \quad \vartheta_{2}=p^{3} z, \quad \vartheta_{3}=p^{6} z\left(z^{2}-h^{2}\right)$, $\vartheta_{4}=p^{5}\left(z^{2}-h^{2}\right), \quad \vartheta_{5}=p^{3}$.

Соответствующие коэффициенты $a_{s t}$ и $a_{s t}^{\prime}$ будут

$$
a_{s t}=d_{s} d_{t} b_{s t}, \quad a_{s t}^{\prime}=d_{s} d_{t} b_{s t}^{\prime},
$$

где

$$
\begin{gathered}
b_{11}=2 p^{4} h, \quad b_{15}=2 p^{5} h, \quad b_{22}=\frac{4}{3} p^{6} h^{3}, \\
b_{23}=-\frac{4}{15} p^{9} h^{5}, \quad b_{33}=\frac{16}{105} p^{12} h^{7} \\
b_{45}=b_{24}^{\prime}=-b_{42}^{\prime}=-\frac{4}{3} p^{8} h^{3}, \quad b_{55}=2 p^{6} h, \\
b_{31}^{\prime}=-b_{13}^{\prime}=-\frac{4}{15} \frac{\lambda}{\lambda+2 \mu} p^{10} h^{6} .
\end{gathered}
$$

Вводя эти коэффициенты в уравнения равновесия (1.5) и в соотношения (1.6), видим, что членами $a_{31} \tau^{\alpha}$ и $\mu a_{13}^{\prime} \varphi^{\alpha}$ как высшего порядка малыми нужно пренебречь.

Если вычислять коэффициенты $k_{s}$ по формулам (3.5) и (1.11) и подставлять в уравнения (1.12) и (1.15), получаем уравнения пластинки

$$
\begin{gathered}
\left\{\left[(\lambda+2 \mu) \nabla^{2}-\varrho \partial_{t}^{2}\right]\left[\frac{7}{10} \mu \nabla^{2}-\varrho \partial_{t}^{2}\right]-12 \mu(\lambda+\mu) h^{-2} \nabla^{2}+\right. \\
\left.+3(\lambda+2 \mu) h^{-2} \varrho \partial_{t}^{2}\right\} w=0 \\
\left(\mu \nabla^{2}-\varrho \partial_{t}^{2}\right) \Phi=0 .
\end{gathered}
$$

Уравнения (3.6) отличаются от соответствующих уравнений пластинки шестого порядка, выведенных Кэйном-Миндлином [6], тем, что имеют множитель $\frac{7}{10}$ перед оператором $\mu \nabla^{2}$. Это объясняется обстоятельством, что уравнения Кэйна-Миндлина неправильно учитывают распределение напряжений $\sigma^{i 3}$ по толщине піластинки. 


\title{
Л И ТЕРА Т У Р A
}

1. Я. С. У флянд, Распространение волн при поперечных колебаниях стержней и пластин, ПММ, т. 12, вып. 3, 1948.

2. R. D. Mindilin, Influence of rotatory inertia and shear on flexural motion of isotropic elastic plates, J. Appl. Mech., 18, 1, 1951.

3. V. T. B u chw a ld, Low frequency flexural vibrations in elastic plates, Quart. J. Mech. Appl. Math., vol. 12, Part 4, 1959.

4. E. Volter ra, Influenza del taglio nella dinamica e nella statica delle piastre sottili, Atti Accad. Naz. Lincei. Rend., Cl. Sci. fis. mat. e natur., vol. 28, Fasc. 6, vol. 29. Fasc. $1 / 2,1960$.

5. И. Т. Селезов, Нсследование поперечных колебаний пластинки, Прикладн. мех., т. 6 , вып. 3, 1960 .

6. T.R. Kane, R. D. Mindlin, High-frequency extensional vibrations of plates, J. Appl. Mech., 23, 2, 1956.

7. E. Volterra, E. C. Z a ch $\mathrm{m}$ a nog lou, On longitudinal waves in an elastic plate, Proc. Amer. Soc. Civ. Eng., EM 1, 1896, Jan. 1959.

8. R. D. Mindli n, M. A. M e dick, Extensional vibrations of elastic plates, J. Appl. Mech., 26, 4, 1959.

Ннститит кибернетики

Академии наук Эстонской ССР
Поступила в редакшию

12 VII 1962

\section{ELASTSETE PLAATIDE ARVUTUSMUDELITEST DUNAAMIKAULESANNETE LAHENDAMISEKS}

\author{
L. Ainola,
}

füüsika-matemaatikateaduste kandidaat

\section{Resümee}

Antakse plaatide nn. täpsustatud teooria teatav üldistus. Variatsiooniprintsiipi (1.1). ja aproksimatsiooni (1.2) kasutades tuletatakse kuuendat järku plaadivōrrandid üldisel kujul (1.12) ja (1.15). Näitena vaadeldakse juhtu, kus ümberpaigutused ja pinged on aproksimeeritud elastsusteooria elementaarlahendi kujul (2.2) (antisümmeetriliste ülesannete jaoks) ja (3.1) (sümmeetriliste ülesannete jaoks). Erijuhul, kasutades elementaarlahendit, mis vastab lōpmatult pikkade lainete levikule esimese moodi järgi, saadakseplaadivôrrandid (2.9) ja (3.6), mis tuntud võrranditest erinevad koefitsientide väärtustepoolest.

\section{Eesti NSV Teaduste Akadeemia} Küberneetika Instituut
Saabus toimetusse 12. VII 1962

\section{ON THEORIES OF ELASTIC PLATES FOR DYNAMIC PROBLEMS}

\section{Ainola}

\section{Summary}

A generalization of plate theories of the sixth order is developed. From the variational principle (1.1), by using approximation (1.2), equations of plates in general form (1.12) and (1.15) are obtained. Further the case of approximation in the form of a particular solution of the linear theory of elasticity (2.2) and (3.1) is considered.

In case the displacements and stresses are approximated by particular solution: corresponding to propagation of the elementary waves of infinite wavelength by the first mode of propagation, equations (2.9) and (3.6) are obtained. These equations differ froms the known equations of plates by the values of coefficients. 